\title{
FUNCTIONAL AND PROGNOSTIC ANALYSIS OF AGR2 (ANTERIOR GRADIENT PROTEIN 2) IN BREAST CANCER USING IN-SILIC DATA
}

\author{
Carolina L. Moraes ${ }^{1,2}$, Natália C. Melo33, Tiphany C. de Bessa4 ${ }^{4}$, Waldemar N. do Amaral1,2 \\ ${ }^{1}$ Department of Gynaecology and Obstetrics, School of Medicine, Universidade Federal de Goiás - Goiânia (GO), Brazil. \\ ${ }^{2}$ Graduate Program in Health Sciences, School of Medicine, Universidade Federal de Goiás - Goiânia (GO), Brazil. \\ ${ }^{3}$ Department of Gynaecology and Obstetrics, School of Medicine, Universidade Federal de São Paulo - São Paulo (SP), Brazil. \\ ${ }^{4}$ Department of Cardiology, School of Medicine, Universidade de São Paulo - São Paulo (SP), Brazil.
}

Objective: To characterize the expression profile and the prognostic value of AGR2 in breast cancer and the possible genetic and epigenetic mechanisms involved. Methodology: The METHHC platform was used to evaluate methylation using the TCGA (The Cancer Genome Atlas) data. The gene expression data were extracted from the TCGA and METABRIC databases, which were accessed through cBioPortal. The KMPlotter software was implemented to analyze the overall survival time (OS) and disease-free survival (SLD). Results: The methylation of AGR2 was reduced in the tumor compared to the normal samples $(\mathrm{p}<0.0001)$. Correlation in tumor samples $(\mathrm{R}=-0.5864$; $\mathrm{p}<0.0001)$ and normal $(\mathrm{R}=-0.8333$; $\mathrm{p}<0.0001)$ confirmed the mechanism between methylation and gene expression. Increased AGR2 expression was associated with positivy of estrogen receptor $(p \leq 0.0001)$ and progesterone $(p \leq 0.0001)$, HER 2 negativity $(p \leq 0.05)$ and the post menopause $(p=0.0001)$ in the data of METABRIC and TCGA. AGR2 expression was reduced in the basal and claudin low subtypes, and increased in luminal subtypes A and B, HER2 and the normal-like subtype, with $\mathrm{p} \leq 0.0001$ in the METABRIC data. Low AGR 2 expression was associated with increased histological grade $(\mathrm{p} \leq 0.0001)$ and cancer staging $(\mathrm{p}=0.0476)$. Reduction of AGR2 expression conferred worse SG in the luminal subtype A ( $\mathrm{p}=0.0027)$ and the worse SLD in the basal subtype $(\mathrm{p}=0.00027)$. Meanwhile, the high expression of AGR2 conferred worse SG ( $p=0.018)$ and worse SLD ( $p=0.0018)$ in patients of the B luminal subtype. Conclusion: According to our findings, AGR2 is hypomethylated in breast cancer. Its modulation is associated with progression of breast cancer and is related to established markers. In addition, AGR2 gene expression analysis appears to be an important biomarker in the identification of tumor subtypes and has prognostic value. 